\title{
Implementation effectiveness of health interventions for indigenous communities: a systematic review
}

Truely Harding ${ }^{*}$ (D) and John Oetzel(D)

\begin{abstract}
Background: Translating research into practice is an important issue for implementing health interventions effectively for Indigenous communities. He Pikinga Waiora (HPW) is a recent implementation framework that provides a strong foundation for designing and implementing health interventions in Indigenous communities for non-communicable diseases around community engagement, culture-centred approach, systems thinking and integrated knowledge translation. This study addresses the following research question: How are the elements of the HPW Implementation Framework reflected in studies involving the implementation of a non-communicable disease health intervention in an Indigenous community?

Methods: A systematic review was conducted using multiple databases. Studies were included if they involved the implementation or evaluation of a health intervention targeting non-communicable diseases for Indigenous communities in Australia, Canada, New Zealand or the United States of America. Published quantitative and qualitative literature from 2008 to 2018 were included. Methodological appraisal of the included articles was completed using the Joanna Briggs Institute System for the Unified Management, Assessment and Review of Information. Data on the population, topic, methods, and outcomes were detailed for each individual study. Key data extracted included the HPW elements along with study characteristics, who delivered the intervention and health outcomes. Data analysis involved a qualitative synthesis of findings as guided by a coding scheme of the HPW elements.
\end{abstract}

Results: Twenty-one studies were included. Health topics included diabetes, nutrition, weight loss, cancer and general health. The key themes were as follows: (a) two thirds of studies demonstrated high levels of community engagement; (b) from the culture-centred approach, two-thirds of studies reflected moderate to high levels of community voice/ agency although only a third of the studies included structural changes and researcher reflexivity; (c) about a quarter of studies included multi-level outcomes and activities consistent with systems thinking, 40\% had individual-level outcomes with some systems thinking, and 33\% included individual-level outcomes and limited systems thinking; and (d) almost $40 \%$ of studies included high levels of end user (e.g., policy makers and tribal leaders) engagement reflective of integrated knowledge translation, but nearly half had limited end-user engagement.

Conclusions: The HPW Implementation Framework is a comprehensive model for potentially understanding implementation effectiveness in Indigenous communities. The review suggests that the studies are reflective of high levels of community engagement and culture-centredness. The long-term sustainability and translation of evidence to practice may be inhibited because of lower levels of systems thinking and integrated knowledge translation.

Registration: Not registered

Keywords: Indigenous communities, Health interventions, Non-communicable diseases, Implementation framework, Community engagement

\footnotetext{
* Correspondence: truely.harding@gmail.com

Waikato Management School, University of Waikato, Private Bag 3105,

Hamilton 3240, New Zealand
}

C) The Author(s). 2019 Open Access This article is distributed under the terms of the Creative Commons Attribution 4.0 International License (http://creativecommons.org/licenses/by/4.0/), which permits unrestricted use, distribution, and reproduction in any medium, provided you give appropriate credit to the original author(s) and the source, provide a link to the Creative Commons license, and indicate if changes were made. The Creative Commons Public Domain Dedication waiver (http://creativecommons.org/publicdomain/zero/1.0/) applies to the data made available in this article, unless otherwise stated. 


\section{Background}

Each year, billions of dollars are spent around the world to support the development of evidence-based health interventions for non-communicable diseases designed to improve human health and reduce health inequities [1, 2 ]. Only a small fraction of these interventions are ever successfully implemented into practice [3], and efforts to implement these practices can take many years [4]. The translation of evidence-based guidelines into practice is one of the most challenging problems in health care and disease prevention [5]. Despite extensive public health research on the efficacy and effectiveness of health promotion and disease prevention strategies, methods for disseminating these interventions and encouraging their implementation and wide-spread adoption are not well developed or evaluated [5].

Further, little progress has been made in reducing inequities despite the fact that there is strong evidence supporting intervention effectiveness in regards to noncommunicable diseases [6]. Researchers acknowledge the need for implementation science and translational research for achieving health equity and have identified key issues including context, culture and levels of acceptance as central to the problem of the utilisation of evidence-based practices $[7,8]$. Translation, dissemination, uptake and implementation are becoming increasingly important to transition innovative health research into health policy and practice and ultimately achieve health equity for Indigenous populations $[3,7]$.

Indigenous populations around the globe face inequities compared to non-Indigenous populations $[9,10]$. For example, one study found inequities between Indigenous and non-Indigenous populations in relation to life expectancy, child obesity, adult obesity, educational attainment and economic status [10]. Achieving health equity requires addressing a complex array of contextual and cultural features along with the unjust distribution of social determinants in health rather than simply focusing on intervention efficacy $[7,8]$. For example, Indigenous perspectives on holism and wellbeing are based on cultural values, beliefs and traditions passed down the generations, including beliefs in the unity of mind, body and spirit [11]. Indigenous cultures frequently believe that all life is interrelated including the environment and the universe and that holism is the most appropriate way to understand health and wellbeing [12, 13]. Thus, when implementing an intervention with an Indigenous community, the intervention needs to be culturally appropriate and relevant as well as supported and owned by the community $[2,14]$.

A recent implementation framework provides a strong foundation for understanding the key principles for developing and implementing non-communicable disease health interventions with Indigenous communities. The
He Pikinga Waiora (HPW; Enhancing Wellbeing) [15] is a theoretical framework that fills a gap in regards to the lack of implementation models for Indigenous communities, which may help account for the underwhelming progress made in reducing health inequities [16]. HPW is built on a strong international evidence base for best practice in developing and implementing health interventions [15]. Specifically, it argues that implementation science for Indigenous communities should be grounded in Indigenous knowledge, participatory approaches and systems thinking and includes four elements: culturecentred approach, community engagement, systems thinking and integrated knowledge translation.

First, implementation should be guided by the culturecentred approach (CCA). The CCA argues that social structures of health can be transformed by providing opportunities for community voice/agency, reflexivity among researchers, and providing resources to address structural challenges [17, 18]. This transformation is achieved through asserting Indigenous self-determination, challenging power imbalances and health researchers/professionals being reflexive and adjusting their behaviour to enhance cultural safety $[15,19]$. Such an approach helps to ensure Indigenous cultural perspectives are part of the definition of the problem and integrated into the interventions to facilitate implementation effectiveness and address health equity [20].

Second, high levels of community engagement (CE) are associated with greater implementation effectiveness and improved health outcomes and health equity [21, 22]. CE is a process of collaborating with groups directly affected by a particular health issue or with groups who are working with those affected [23]. CE ranges from very limited community involvement to community ownership and management through five categories: outreach, consultation, involvement, shared leadership and community-driven [24, 25]. High levels of CE are reflected through shared decision-making and communication among researchers and community members which helps with sustainability, capacity building and long-term health outcomes [26, 27].

Third, systems thinking (ST) helps to address the complexity of the local contexts and the variety of levels and determinants of health problems [28, 29]. ST also facilitates new framings and strategies that are associated with improved project and health outcomes including health equity $[29,30]$. It allows for new ways of thinking for researchers, practitioners and community members through considering different perspectives, relationships among people/facets of the health system and multiple level of analysis [30]. ST also acknowledges holistic perspectives towards health problems and examines the inter-relationships of the various parts that need to be understood within a larger context [29]. It is important to note that 
ST is frequently used and has many different approaches to conceptualising such as complex systems dynamics. As a result, there are no clear guidelines for implementation in practice [31, 32]. The HPW framework specifies key ST elements that may serve as guidelines for implementation of health interventions for Indigenous communities including multiple perspectives, relationships and levels of analysis along with feedback loops.

Finally, integrated knowledge translation (IKT) emphasises co-design and co-production with end users in developing and implementing an intervention for the purpose of transferring knowledge and enhancing sustainability [33, 34]. End users are the people who will use research findings and facilitate the translation from research to practice [35]. These may be clinicians, policy makers, tribal leaders and systems administrators. IKT involves the researchers and end users working in various levels of partnership to ensure there is shared ownership and that many barriers to implementation and translation can be addressed early in the design process $[33,36]$. For Indigenous communities especially, IKT also needs to ensure there is benefit for the community reflected in the knowledge of the community [37].

The purpose of this study is to conduct a systematic literature review of articles that involved the implementation of a non-communicable disease health intervention in an Indigenous community. Systematically reviewing the literature will provide insights regarding how the HPW principles are currently being implemented and reported in Indigenous community-based health interventions. This study applies the HPW framework in a post hoc manner to identify the patterns in intervention development and implementation with Indigenous communities.

\section{Methods}

The systematic review was completed using PRISMA guidelines [38] (see Additional file 1 for the checklist). Our primary research question was the following: How are the four elements of the HPW Implementation Framework reflected in studies involving the implementation of a non-communicable disease health intervention in an Indigenous community? This question relies on the post hoc application of the HPW framework to studies that did not directly use it. The rationale for this choice is that there is not an existing framework guiding implementation science of Indigenous health interventions. The HPW framework was recently developed and has a strong theoretical and empirical basis and its post hoc application enables us to examine whether these key elements are being used by researchers and implementers; if so, they can help identify promising practices for researchers and practitioners working in similar communities. If they are not being used, it may illustrate important directions for future research and practice. The
HPW framework, previously applied in a post hoc manner, provided insights demonstrating associations between the implementation of framework principles and health outcomes in type 2 diabetes prevention for Indigenous people in primary care [15].

\section{Inclusion and exclusion criteria}

The chosen literature was peer reviewed and published in English since 2008. This time period was selected to provide relatively recent insights to implementation effectiveness and provide a sufficient literature base to review. Literature was only considered if it evaluated and/ or implemented a health intervention targeting Indigenous communities. Communities are physical spaces involving Indigenous members who were targeted for benefit from the health intervention. The specific interventions included in this study were those that discussed non-communicable diseases.

The search exclusion criteria eliminated articles that were reviews or editorials. Further, the article was excluded if the intervention took place in a primary health organisation or was based on another aspect of the primary health system. Additionally, school-based interventions were excluded unless the school-based intervention was part of a larger implementation into the community (e.g., involving larger health promotion and community intervention). Literature was excluded if the study population was not Indigenous and if there was no intervention implemented. Literature was also excluded if it only discussed the process of creating and implementing an intervention rather than evaluation of the intervention process and/or outcomes.

\section{Search strategy}

EBSCOhost, Emerald Insight, ProQuest Central, Pubmed and MEDLINE databases were the selected search engines. The key search terms were community health, Māori, First Nation, Aboriginal, Native American, Indigenous and intervention. The search consisted of combining two or three search terms to reveal specific articles that were relevant to the study. The following sequences were the search combinations used for this study: "community health" and "Māori"; "community health" and "Māori" and "intervention"; "community health" and "Indigenous"; "community health" and "Indigenous" and "intervention"; "community health" and "First Nation"; and "community health" and "First Nation" and "intervention"; "community health" and "Aboriginal"; and "community health" and "Aboriginal" and "intervention"; and "community health" and "Native American"; and "community health" and "Native American" and "intervention". The terms were searched in the article title, abstract, the whole article and the keywords. Literature from each individual search was exported to 
an EndNote file to identify and eliminate the duplicate articles. Once the duplicates were removed, the study selection process began.

Study selection was completed by the two authors. Titles and abstracts were completed by the first author with consultation with the second if there were uncertainties. The full-text articles were independently reviewed by both authors using the exclusion criteria. After completing the study selection, additional records were identified using three means to locate any missed published or unpublished studies and thus reduce the risk of publication bias [39]. First, a manual search of references from the included articles was undertaken. Second, a search of the grey literature was completed using the search terms in Google. Third, three clinical trial registries (Australia New Zealand Clinical Trials Registry, Health Canada Clinical Trials Database and ClinicalTrials.gov) were searched using key Indigenousrelated search terms (e.g., First Nations). Relevant trial descriptions were reviewed; study protocol articles and project names were then searched through Google and Google Scholar to find final study results in published or unpublished form.

\section{Data extraction and methodological appraisal}

From the articles that met the inclusion criteria, the selected data for this study were the population, health topic, methods, measures, outcome(s) of the health intervention, who delivered the intervention and data related to the HPW elements. Given that we are using the HPW framework as a post hoc analysis of the articles and also that some published outcome studies have limited information about intervention development, we also extracted data from cited studies in the included articles such as study protocols, supplemental files, web sites or articles that were referenced to provide more information about the study methods or intervention. In two cases, a follow-up publication identified in study selection helped provide additional information about the primary study [40, 41]. We assigned a rating of the quality of details provided in the articles as good, fair or poor. In all cases, the rating was at least fair with the vast majority rated as good $(n=17)$ which allows the comprehensive assessment of each of the HPW elements (see Additional file 2 for information about additional studies consulted and quality of details).

Methodological appraisal of the included articles was completed using the Joanna Briggs Institute System for the Unified Management, Assessment and Review of Information [42]. The study design for the primary hypotheses/study aims were categorised along with three different types: observational, randomised control trials and qualitative. Each individual study was assessed with risk of bias identified. Appraisal criteria are displayed in
Table 1. Each criterion was rated as yes, somewhat, no, unknown or not applicable. An overall score was provided for each study using yes $=2$, somewhat $=1$ and no or unknown $=0$.

\section{Data synthesis}

Summary tables were provided for the primary research question and also for the study characteristics. Data about the HPW elements were described using a coding scheme to guide in the inclusion of key elements [15]. The coding scheme was used to recognise key HPW concepts even if they were not directly labelled as such by the study authors (i.e., current authors' interpretation of whether HPW elements were used). The key components for each of the elements include the following: (a) $\mathrm{CCA}-$ community voice/agency in defining problem and solution/intervention, researcher reflexivity and resources for structural change; (b) $\mathrm{CE}$-degree of shared decision-making and communication among researchers and community entities; (c) ST-multiple perspectives of causes reflecting holism, complex relationships among ideas and entities and multiple levels of analysis (e.g., micro, meso and macro); and (d) IKT-co-design and implementation of intervention with end users. Data were then qualitatively synthesised to provide an overview of how each of the four HPW elements was reflected in the 21 studies.

Data on the population, topic, methods, and outcomes were detailed for each individual study. Individual study results include measures of change and significance values. Given the heterogeneity of study characteristics, measures and outcomes, meta-analysis-or even simple quantitative associations-was not possible. Following a previous systematic review, outcomes were categorised in two ways: (a) having at least one statistically significant change in a primary outcome and (b) having statistically significant changes in $50 \%$ or more of the primary outcomes [43]. Additional file 3 presents the study characteristics and outcomes.

\section{Results \\ Study selection}

Figure 1 shows the search strategy identified 6981 articles from the listed databases; articles were downloaded to EndNote to remove a total of 3590 duplicates. Upon screening for inclusion criteria in titles and abstracts, the full text of 86 articles was independently reviewed. After review using the exclusion criteria, 19 articles were included for analysis. The additional record search resulted in two additional articles for a final total of 21 .

\section{Methodological appraisal}

Table 1 provides a summary of the results of the appraisal. There were seven observational studies: four from the 


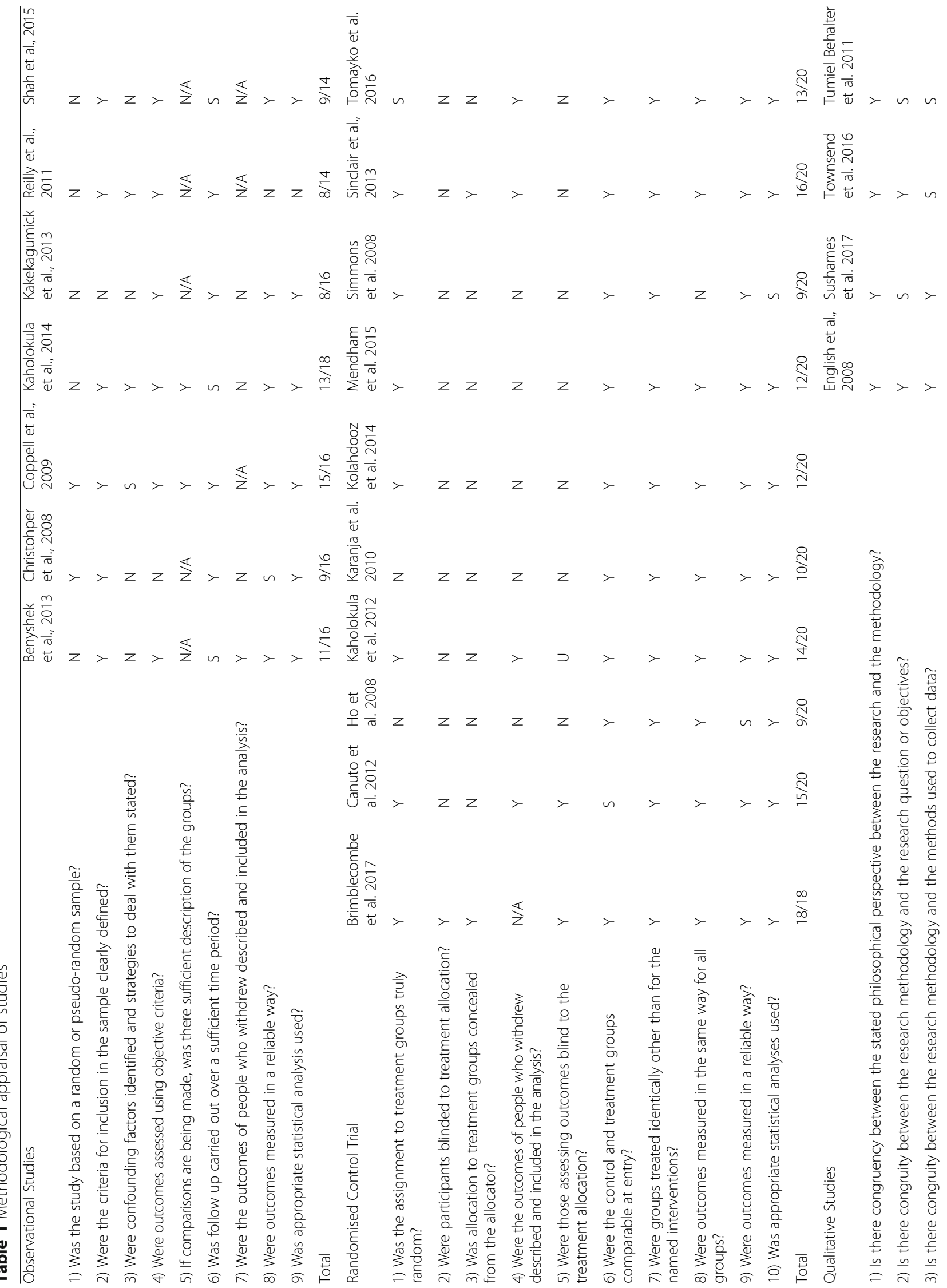




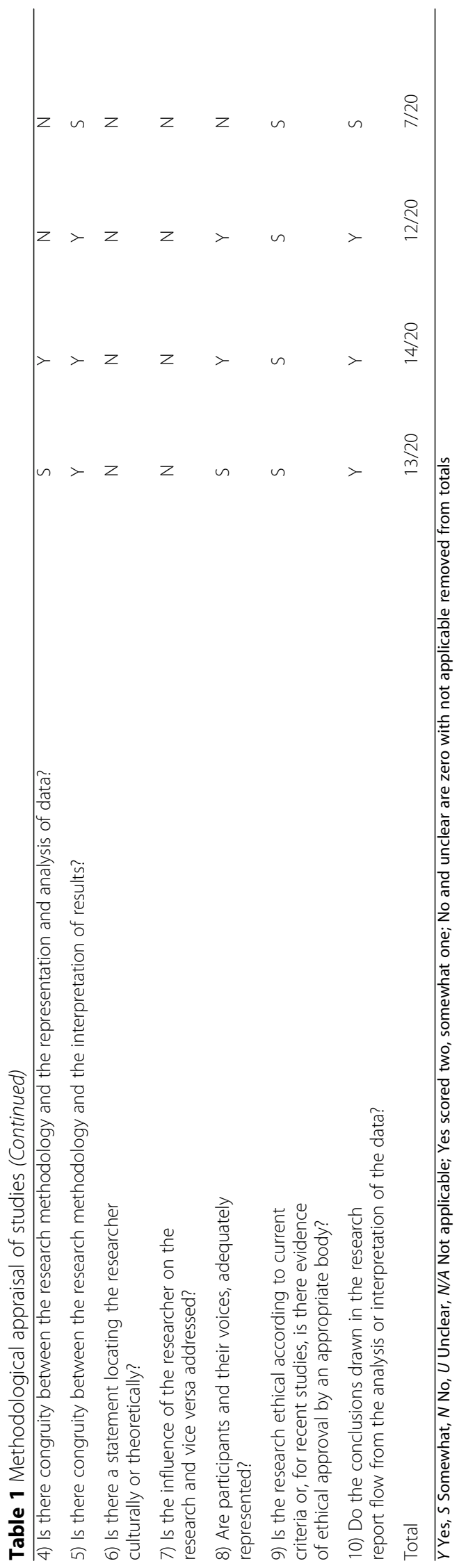




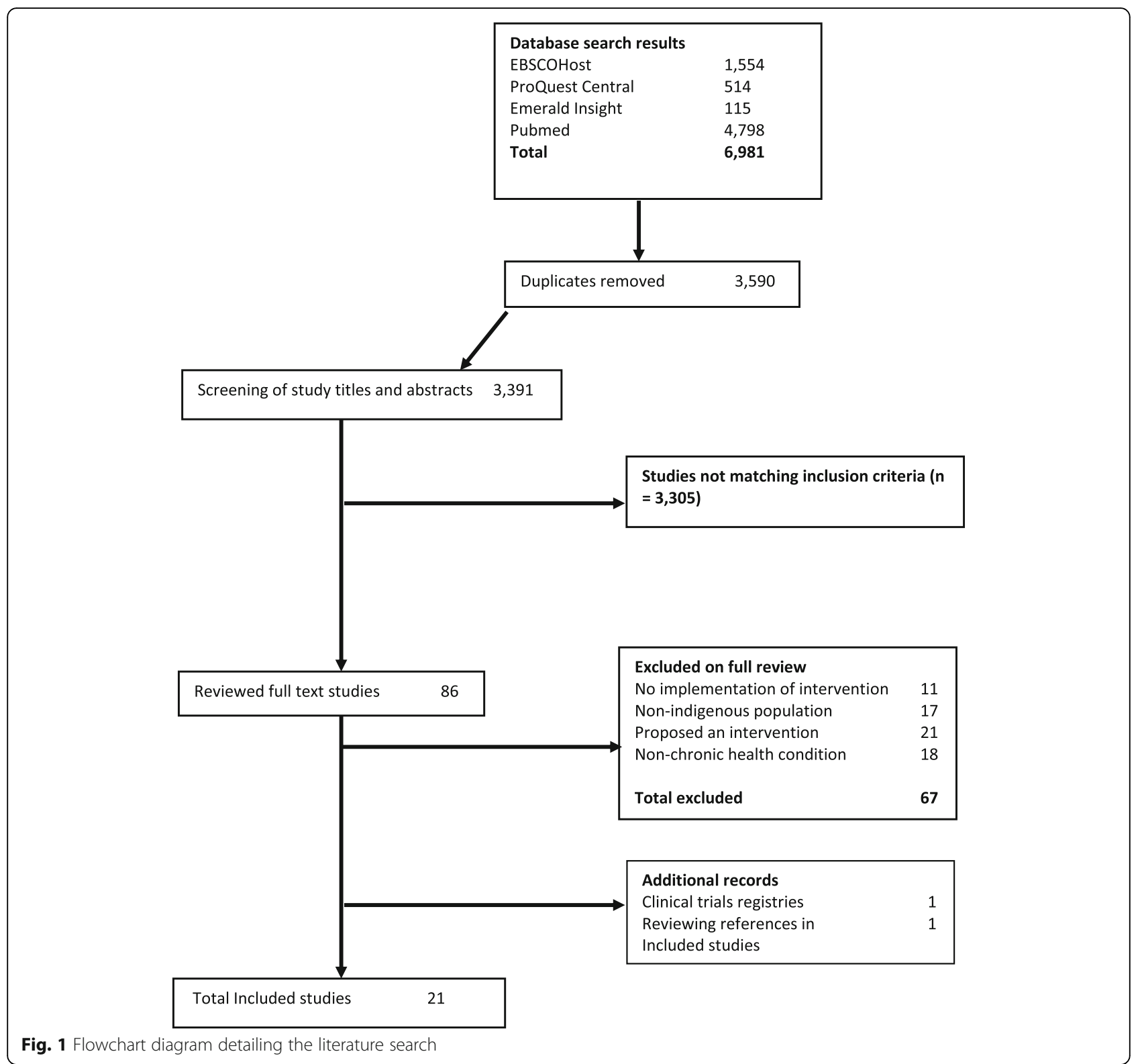

United States of America (USA) [44-47], one from Australia [48], one from New Zealand [49] and one from Canada [50]. The follow-up period ranged from 4 months to 3 years. Three studies had equal or less than 6 months of follow-up which is a potential area for bias [44, 46, 47]. Retention rates ranged from $55-100 \%$ although one study included two independent panels [49] and another did not use individuals as the unit of analysis [48]. The major risk of bias in these studies is the lack of a comparison group although this is consistent with the research design. Additional risks include some lack of valid and reliable measures [45, 48], small sample size [44, 50], lack of appropriate statistical analysis [48], incomplete description of the participants and study methods [50] and lack of information about non- completers [46, 50]. The average study quality rating of observational studies was $68.71 \%(\mathrm{SD}=15.38)$.

There were 10 randomised control trials with comparison groups: four from the USA [51-54], three from Australia [55-57], two from Canada $[58,59]$ and one from New Zealand [60]. Six of the studies randomised individuals to intervention and control/standard care $[51,52,54,56,57,60]$, three included random selection of communities to intervention and control $[53,55,59]$ and one included selection of communities as well, but it was not clear whether assignment was random [58]. In all but two of the studies, the control group received a delayed intervention [53, 60]. The trial periods ranged from 3-24 months (median 9-12 months) with four studies having less than or equal to 6 months of follow- 
up [51, 52, 56, 57]. Four studies reported lower than $70 \%$ retention rate $(59-66 \%)[54,56,57,60]$. The major risks of bias included lack of blinding in randomisation in all but two studies [51, 55], lack of blinding for assessors in all but two studies [55, 56], lack of inclusion of data from those who withdrew (e.g., intention to treat analysis) in all but four studies [51, $52,54,56]$, incomplete reporting of study results in one study [60], some unreliable measures in one study [58] and a few participants moving from one arm to another post-randomisation in one study [54]. The average study quality rating of randomised control trials was $65.00 \%(\mathrm{SD}=17.16)$.

There were four qualitative studies: three from the USA [61-63] and one from Australia [64]. Each of these studies described a health intervention and sought to describe the processes by which the programme was developed and how it impacted outcomes [61-63] or how it related to participation in the intervention [62, 64]. Only two studies directly addressed participant outcomes although those included only descriptive information [61, 62]. A risk of bias is that none of the studies included statements of researcher positionality nor did they discuss the influence of the researcher on the research. Further, two of the studies did not provide any direct quotes thus a risk of bias in that participant perspectives were not included $[62,63]$. The average study quality rating of qualitative studies was $57.50 \%(\mathrm{SD}=15.55)$.

\section{Study synthesis}

The study synthesis addresses the research question about how the HPW elements are reflected in the studies (see Table 2 for the study synthesis). Prior to summarising those findings, Additional file 3 provides a breakdown of the study characteristics that helps to understand the context of the studies. The targeted health conditions included diabetes (48\%), obesity/general non-communicable health conditions (24\%), nutrition (19\%) and cancer (10\%). The types of interventions included lifestyle (38\%), multi-pronged including individual and community elements (33\%), selfmanagement of a condition (14\%) and education (14\%). Two thirds of the interventions included the delivery of at least one component by a community health worker $(\mathrm{CHW})$. All of the studies that had a measurable outcome variable $(n=19)$ had at least one primary outcome with a statistically significant and improved change with six studies $(38 \%)$ achieving significant change in $50 \%$ of primary outcomes measures. A slight majority of the studies (52\%) were feasibility, pilot or short-term interventions.

For the CCA, there are three key issues to consider: voice/agency, reflexivity and structural change and resources. For voice/agency, there were three patterns identified. The first was studies that reflected community voice/agency in defining the problem and identifying the solution $[45,46,48-50,52,54,59,61]$. The second was studies that allowed for adaptation of the solution to fit the culture of the community, but without clear choice that this was an important problem to address $[51,53,56$, $58,62,63]$. The third was studies that did not allow much input into the problem or solution beyond minor changes or simple approval $[44,47,55,57,60,64]$. Reflexivity of the researchers about power relations and relationships among partners was directly expressed by a little more than $40 \%$ of the studies [ $45,46,48,49,51,52,61,62]$, although an additional study did include post-study reflection [60]. Finally, while all of the studies offered resources, only a third of the studies sought structural changes through their interventions in the form of changing policies, systems or organisational/community practice $[45$, $48-50,58,59,61]$.

High levels of CE were reflected in two thirds of the studies. The most common engagement approach was the use of community-based participatory research (CBPR) which was directly noted by nine of the studies [44-46, 51, 52, 54, 59, 61, 62] with the remaining studies offering another participatory approach [48-50, 53, 63]. Most of these studies involved community partners or steering/advisory groups that guided the work and had shared decision-making and communication responsibilities with the researchers. There was evidence in these studies that the high level of engagement was included throughout the research process from design/adaptation to implementation and evaluation of the intervention. The remaining studies had relatively limited levels of engagement. Some of these would be best described as an initial consultation to get approval for the project with limited input beyond that stage except to help with recruiting participants $[47,55,57]$. Two of these limited engagement studies stated the use of steering/advisory committees to guide the work and yet the evidence is that these groups were primary for consultation and not shared decision-making $[56,60]$. Three additional studies stated they used participatory approaches although with limited evidence of who the partners were or how the studies were in fact participatory $[44,58,64]$. Finally, some of these limited engagement studies utilised CHWs to help with engagement with participants even though other aspects of their project were limited engagement [44, 47,60].

There were three predominant patterns of ST, which in part are based on the level of behaviour targeted and in part on the perspectives and relationships identified. The vast majority of studies targeted individual behaviour $(n=$ 16; 76\%) with both community and individual-levels targeted by three studies $[49,50,63]$ and the communitylevel only in two studies $[48,55]$. The studies that targeted some community-level behaviour represent the first 


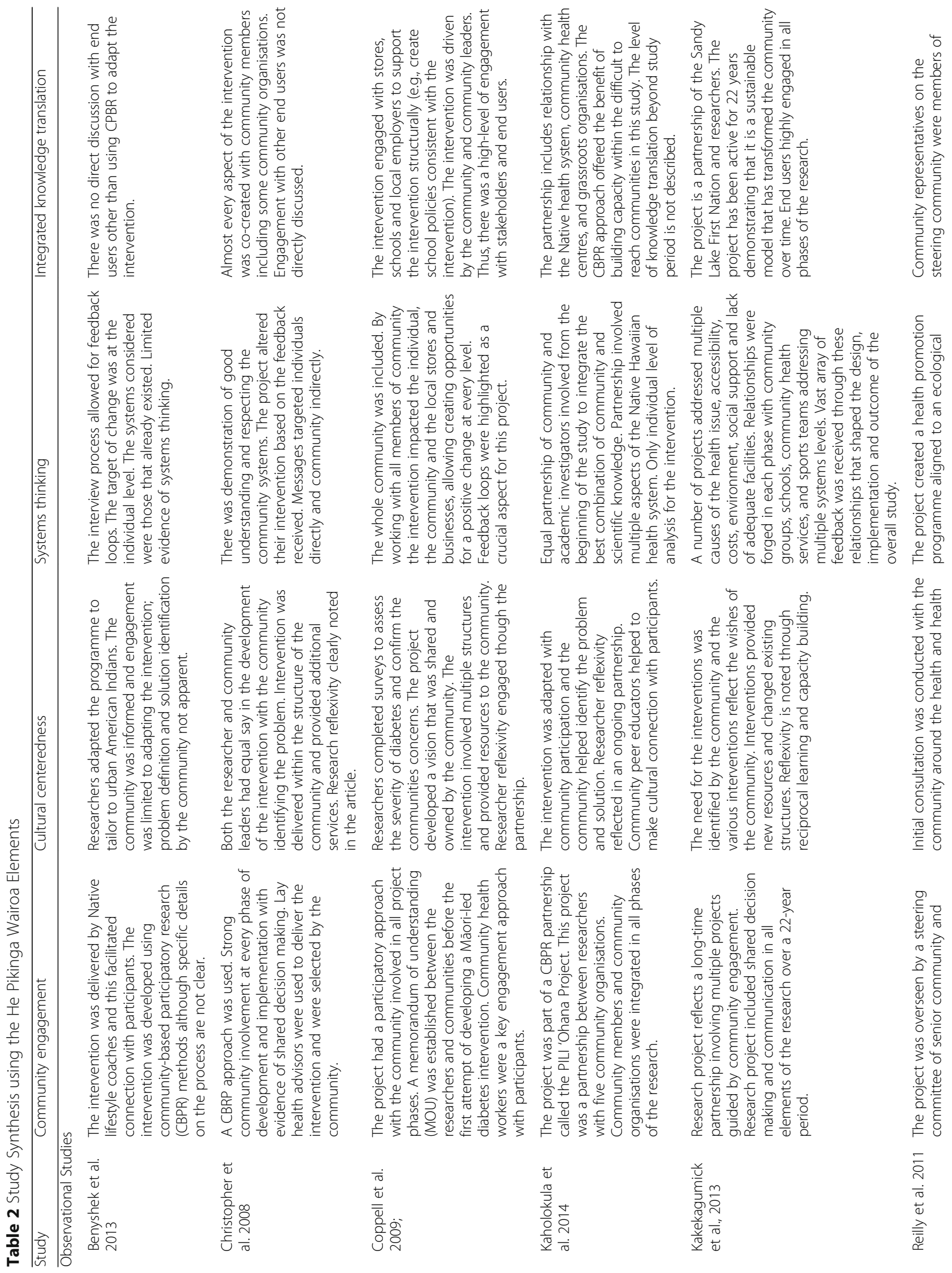




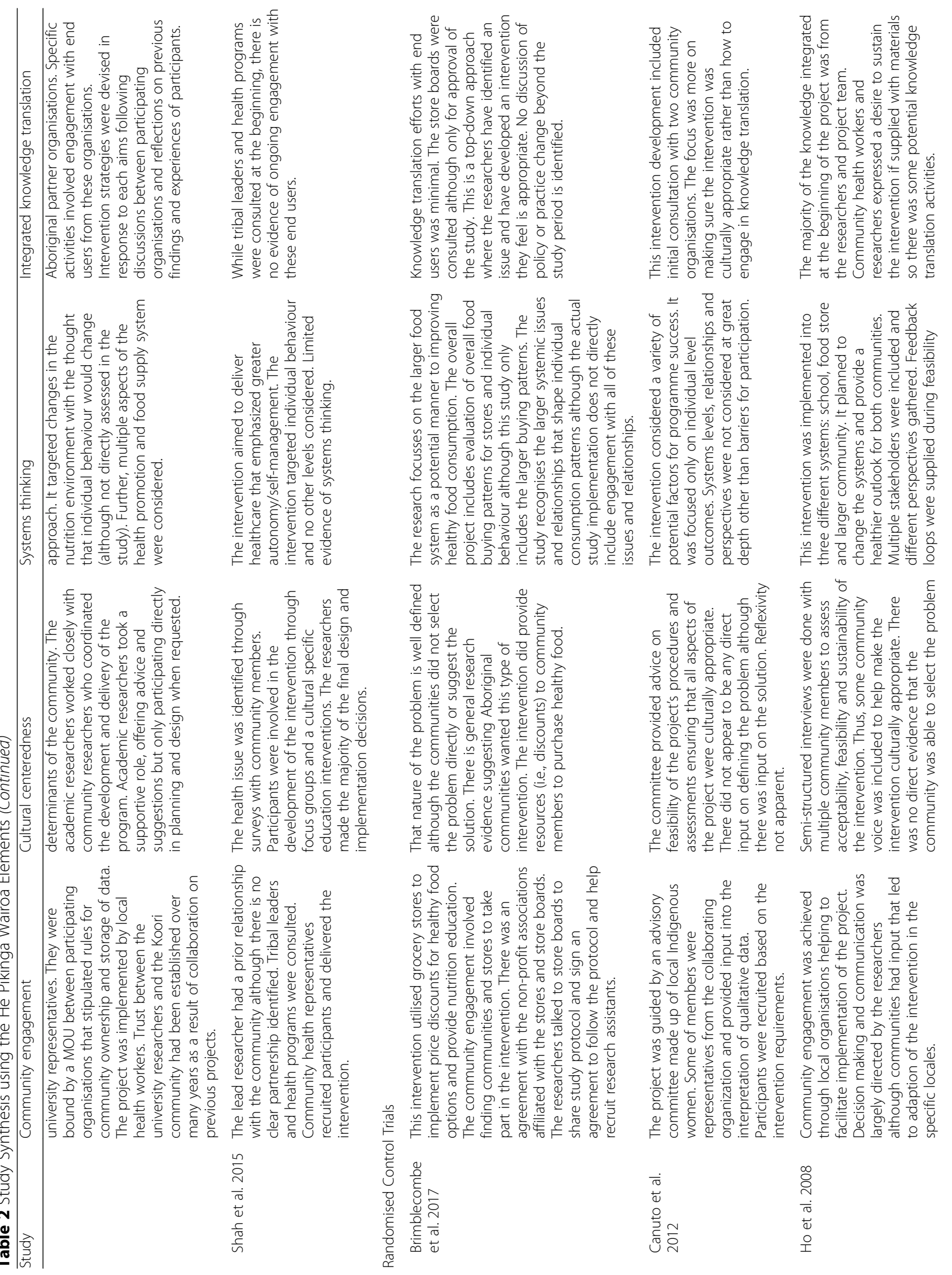




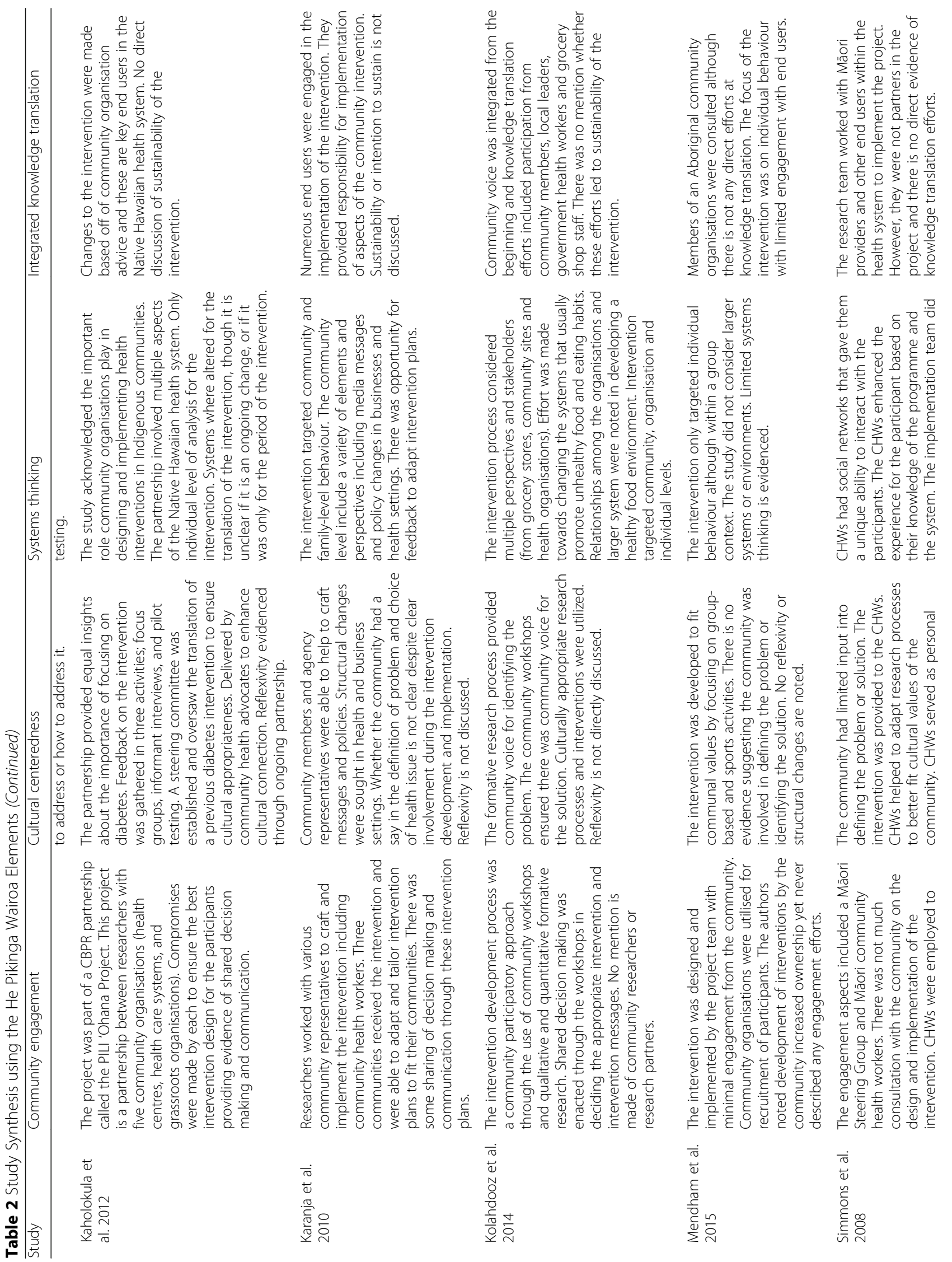




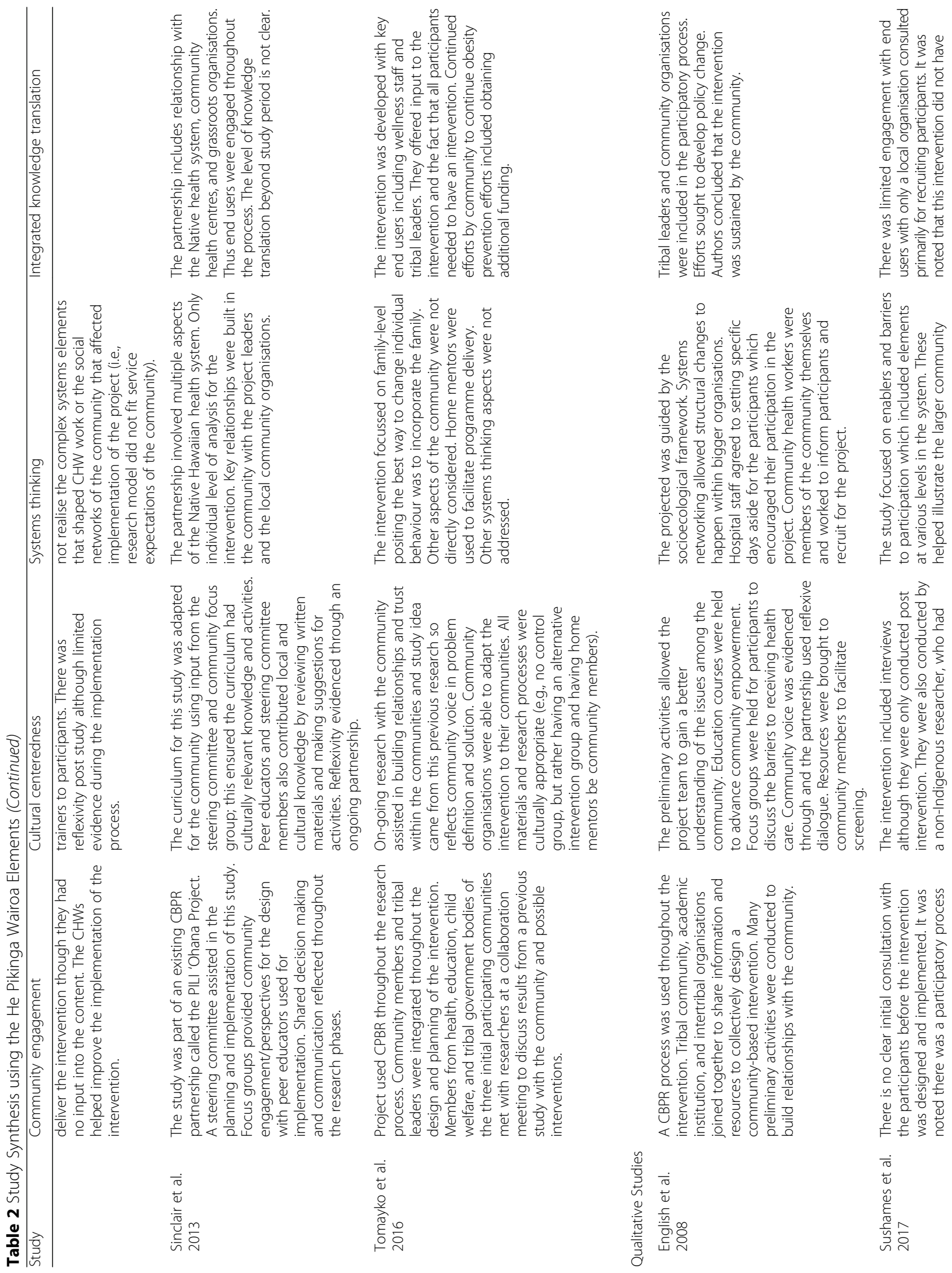




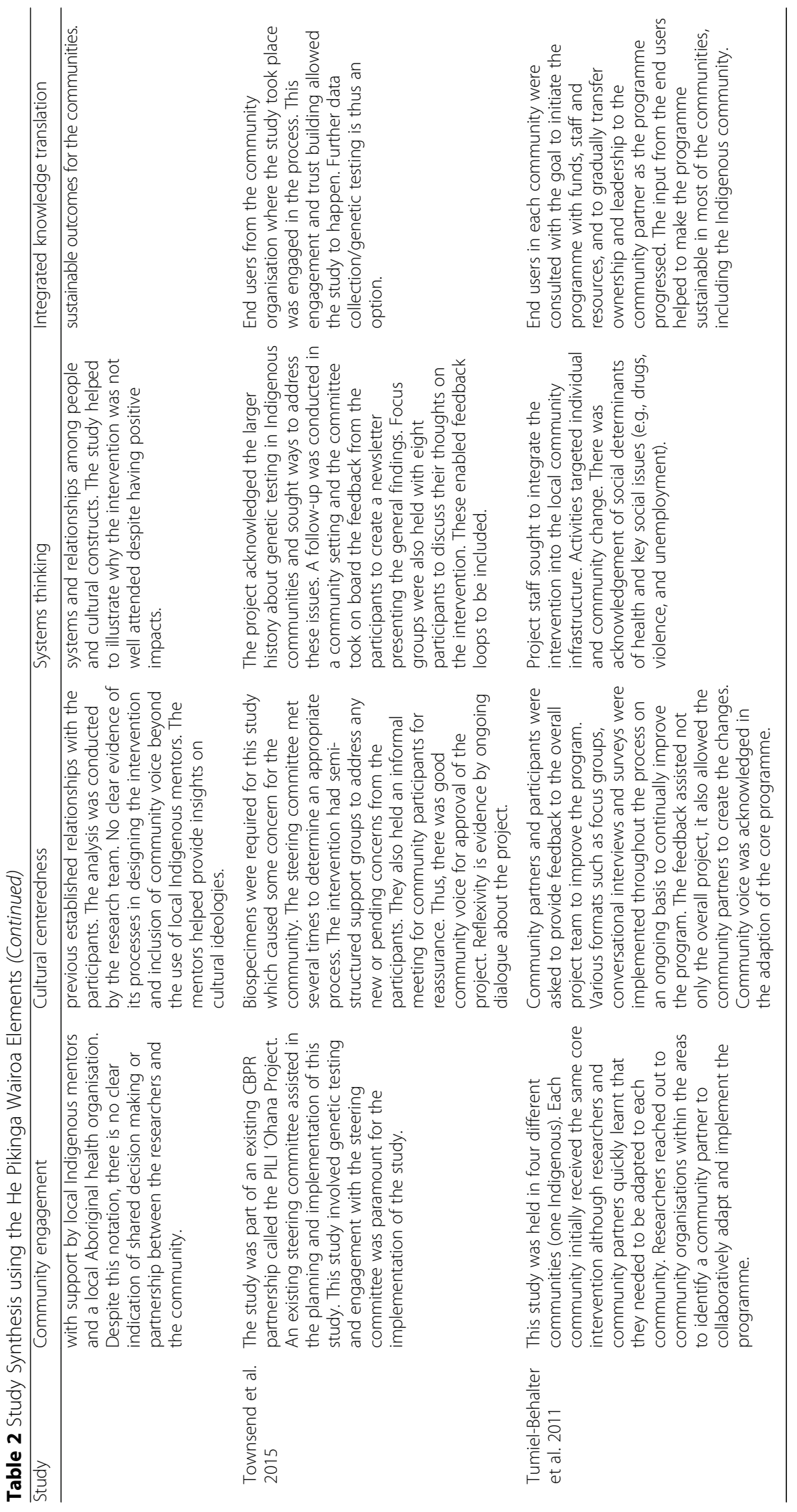


pattern. Each of these studies demonstrated clear understanding of multiple causes and perspectives and included systems-level activities. Most of these studies also had multi-level intervention activities as well. The second pattern was studies that focussed only on individual-level behaviour and demonstrated limited ST [44, 47, 54, 56, 57, $60,64]$. These studies did not integrate multiple perspectives and typically only included minimal feedback loops in adapting the intervention. Two of these studies provided retrospective recognition of $\mathrm{ST}$ as providing explanations for the challenges in implementing the intervention [60, 64]. The final pattern were studies that targeted individual-level behaviour although included ST in the design of the intervention. Four of these studies included multi-level activities in the intervention $[53,58,59,61]$, while the others integrated ST through partners and steering committees to help improve implementation effectiveness [45, $46,51,52,62]$.

IKT includes three predominant approaches in the studies. First, nine studies had limited or no knowledge translation activities or engagement with end users [44, $45,47,55-58,60,64]$. These studies may have consulted end users at the beginning of the study although that was primarily for the purpose of approving the study or gaining access to participants. One of these studies did actively engage with end users in knowledge translations at the end of the study although they did not appear to be integrated throughout the study [58]. Second, four studies included end users through a steering committee that included members of the health system $[46,51,52$, 62]. Thus, the end users were integrated into the design and implementation of the intervention; however, these studies did not directly discuss how knowledge translation activities occurred or whether the intervention was sustainable. Third, eight studies described the integration of community and organisational leaders throughout the design and implementation process and also discussed how the study led to continued activities or funding and/or structural or policy changes [48-50, 53, 54, 59, 61, 63]. These studies represent high levels of IKT.

\section{Discussion}

The purpose of this study was to systematically review the implementation of non-communicable disease health interventions into Indigenous communities and identify the degree to which HPW elements were reflected in these studies. The studies demonstrate a number of positive health outcomes at both individual and community levels and cover a range of non-communicable diseases. Two key patterns emerge about the implementation of these interventions: (a) high levels of $\mathrm{CE}$ and $\mathrm{CCA}$-including the prominence of community health workers-and (b) comparatively lower levels of ST and IKT. Implications and limitations are noted.
Community engagement and culturally-centred approach About two thirds of studies identified participatory approaches as being prominent in the design. These findings reflect the extant literature that argues for participatory approaches to developing and implementing health interventions with Indigenous communities [23, 65, 66]. Further, a variety of systematic and meta-analytic reviews have found positive associations between $\mathrm{CE}$ and health outcomes [21, 26, 67] with the most popular CE approach being CBPR. In addition, an international literature review found that $C E$ has been linked to positive outcomes such as social capital and neighbourhood unity for socially excluded groups [21]. This is supported by an evaluation suggesting that interventions led by community organisations were more successful at engaging secluded groups than government initiatives [67].

Similarly, the CCA is consistent with CE as it emphasises community voice/agency for engaging in change around health [17]. Such an approach centres culture and cultural perspectives and thus is consistent with Indigenous autonomy and self-determination. Many Indigenous organisations have placed a priority on the development of an Indigenous health workforce that has both professional and cultural competence [68], drawing on the fact that culturally adapted health interventions are more effective than traditional "top-down" interventions [69]. Beyond the participatory approach, the CCA advocates for reflexivity of external partners and structural change to facilitate implementation effectiveness. These elements reflect the need for interventions to provide resources and systems change to improve health equity [17]. However, only slightly more than a third of the studies had evidence of research reflexivity or structural change within the studies.

A key way that many studies helped to support CE and/or the CCA was the use of CHW. The majority of studies in this review used $\mathrm{CHW}$ although they may have been called lay health advisers, peer educators, or lifestyle coaches. CHW are considered to be successful due to the relationship they have with the community; they are trusted members who are able to communicate effectively with community members because they are aware of cultural values and reflect the diversity of the population served (i.e., they have cultural knowledge) [70]. The prominence of CHW in these studies is consistent with the extant literature finding frequent use of CHW particularly in Indigenous and ethnic minority communities [71-73]. CHW involvement in interventions is associated with a variety of positive health outcomes including non-communicable diseases and benefits to health service utilisation [71, 74]. CHW are also generally part of an overall philosophy that reflects Indigenous knowledge and participatory approaches such as community engagement [75]. However, it is 
important to note that some studies in this review that have lower CE and CCA still used CHW $[47,60]$; hence, the presence of $\mathrm{CHW}$ does not mean an intervention automatically has high levels of CE and CCA.

\section{Systems thinking and integrated knowledge translation}

While the reviewed studies collectively had high levels of $\mathrm{CE}$ and CCA, there were fewer studies with high levels of ST and even fewer with a high level of IKT. For ST, only a small number of studies had multi-level perspectives and activities and focused on outcomes at a systems level. More studies included information reflecting systems perspectives and multi-level activities although focussed only on individual-level outcomes with a third of studies having limited ST. ST helps to identify a holistic perspective of health issues and also provides boundaries of the intervention within the system for effective implementation (e.g., recognising facilitators and barriers) [76]. Recent literature suggests that combining participatory approaches with ST is the key to improving health equity in communities [29]. Participatory approaches enable multiple stakeholders and perspectives consistent with ST; however, the current review included studies with strong participatory approaches without ST [54] and also strong ST without participatory approaches [55].

Slightly more than a third of the studies in this review demonstrated high levels of end-user engagement and thus IKT, while the other studies had limited or only some engagement with end users. IKT is an important factor for facilitating the translation of evidence-based interventions into policy and practice as it helps to navigate larger health systems and the perspectives of key stakeholders [33, 37, 77]. End users often have the power to shape policy and provide resources to sustain interventions; their integrated engagement provides an opportunity for researchers to understand the larger policy and practice context [33]. Co-design of research between Western researchers and Indigenous end users also facilitates effective knowledge translation between Western scientific and Indigenous knowledge systems [37].

\section{Implications}

This review utilised the HPW framework in a post hoc manner to identify patterns in the implementation of chronic condition health interventions in Indigenous communities. Several implications and some future research results from this review. The relatively high levels of CE and CCA are consistent with autonomy and selfdetermination in Indigenous communities. Autonomy has not been handed to the Indigenous communities, but rather it has been demanded by many Indigenous cultures as a rejection of policies of assimilation resulting from colonial histories [68]. Self-determination is a key element for implementation and intervention effectiveness in Indigenous communities [15, 65]. Selfdetermination facilitates acceptability of interventions because it ensures a sense of ownership, cultural relevance and the centring of Indigenous knowledge to the health problem. Self-determination is often achieved through participatory approaches like CBPR because of the shared decision-making in the interventions [78].

Additionally, enhancing ST and IKT may enhance the sustainability of the health interventions. However, there is clearly a need for future research in this area. This review identified a lack of long-term or systems-level outcomes overall. Much of the focus in outcomes was on individual-level behaviour and knowledge changes. Sustainability was often only included in the discussion of the future implications for the research and not within the research project itself; there were certainly exceptions to this with some projects designed around creating an intervention sustainable beyond at least the study period, and these studies reflected high levels of ST and IKT $[49,50]$.

Very few of the reviewed studies demonstrated high levels of all of the HPW elements. What is not clear at this stage is whether a given intervention needs to be strong in each factor to address health equity. The extant literature demonstrates that each element has positive associations with some aspects of health; although it is not clear whether the collective elements are needed to make a significant improvement in health equity. The diversity of health outcomes, from individual knowledge to system-level change, make it difficult to directly compare the value-added for each individual element. Thus, the current state is that this review provides insights about likely avenues to improve implementation effectiveness for achieving gains towards health equity although does not provide direct evidence.

Finally, there are some interesting insights about the methodological appraisal and the study characteristics. Given that these were studies in the community, most of the studies lacked some of the key elements of traditional research design such as strict blinding in randomisation or even formal adherence to randomisation. Further, in all but two of the randomised trials included in the reviews $[53,60]$, the intervention was eventually delivered to the comparison group or an alternative intervention was provided. This approach is common in Indigenous communities as to withhold an intervention is not consistent with collective values and inclusiveness [66]. These approaches are ways to decolonise research methods to ensure the Indigenous knowledge and values are strongly reflected in the research [66]. This type of inclusivity and community benefit likely should be included in methodological appraisals of Indigenous community health interventions. 


\section{Limitations}

This review is not without limitations. First, given the diversity of health issues, study outcomes and levels of analysis, it is not possible to link study outcomes to specific aspects of the HPW framework. Future research can better explore the concrete relationships between elements of the framework and specific health outcomes. Second, while we have attempted to be rigorous in our search strategy, it is possible that relevant studies have not been included, particularly those not formally published. Also, in the search terms, we opted to use "Native American" and not "American Indian;" hence, relevant studies may have been missed. Finally, we acknowledge that our findings and conclusions are based on the data each publication has provided even if they did not label the information as a particular HPW element. The lack of data regarding ST and IKT does not necessarily mean that they did not consider those elements as page limits may limit reporting of some information. However, we encourage researchers to report on all four HPW elements when describing the implementation of health interventions with Indigenous communities to enable knowledge consolidation about these topics and advance thinking about how best to apply these principles for improved implementation and maximum impact.

\section{Conclusion}

In conclusion, the He Pikinga Waiora Implementation Framework posits that participatory approaches such as CE and CCA, along with ST and IKT, are important to utilise when developing health interventions for Indigenous communities in order to achieve health improvement and health equity. This framework reinforces the idea that Indigenous communities will support health interventions that they help to create as it aligns with their cultural views, making the intervention more beneficial and sustainable for the community. The current review illustrates various patterns of each of the HPW elements with CE and CCA more prominent than ST and IKT. The review also illustrates that few studies incorporate all four elements of the HPW framework although future research is needed to determine the value added for each of the elements.

\section{Additional files}

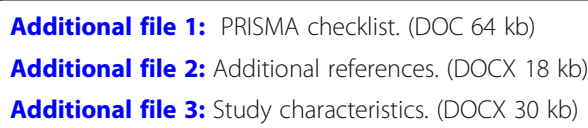

\section{Abbreviations}

CCA: Culture-centred approach; CE: Community engagement; CHW: Community health workers; HPW: He Pikinga Waiora (Enhancing Wellbeing) Implementation Framework; IKT: Integrated knowledge translation; PRISMA: Preferred Reporting Items for Systematic Reviews and Meta-Analyses; ST: Systems thinking; USA: United States of America

\section{Authors' contributions}

$\mathrm{TH}$ completed the systematic review and led the analysis. She led the manuscript writing. JO collaborated on the systematic review and analysis. He supported the writing and editing of the manuscript. Both authors read and approved the final manuscript.

\section{Funding}

This manuscript was supported by a grant from the Healthier Lives National Science Challenge (PI Nina Scott, HL-T1CR-D 13058/1 SUB1320).

\section{Availability of data and materials}

Articles included in the analysis are cited in the reference list.

Ethics approval and consent to participate

Not applicable.

\section{Consent for publication}

Not applicable.

\section{Competing interests}

The authors declare that they have no competing interests.

Received: 8 October 2018 Accepted: 1 July 2019

Published online: 05 August 2019

\section{References}

1. Cooksey D. A review of UK health research funding. London: Her Majesty's Stationery Office; 2006

2. Lines L-A, Jardine $C$. Towards reconciliation efforts: the need for Indigenous voices in Indigenous health strategies. Canadian Public Health Association; 2018. https://www.cpha.ca/towards-reconciliation-efforts-need-indigenousvoices-indigenous-health-strategies. Accessed 15 Sept 2018.

3. Haines A, Kuruvilla S, Borchert M. Bridging the implementation gap between knowledge and action for health. Bull World Health Organ. 2004;82(10):724-31.

4. Chaudoir S, Dugan A, Barr C. Measuring factors affecting implementation of health innovations: a systematic review of structural, organizational, provider, patient, and innovation level measures. Implement Sci. 2013;8:22.

5. Khoury M, Gwinn M, Yoon P, Dowling N, Moore C, Bradley L. The continuum of translation research in genomic medicine: how can we accelerate the appropriate integration of human genome discoveries into health care and disease prevention? Genet Med. 2007;9(10):665-74.

6. Lloyd M, Cook J, Ahmed S, Yonas M, Coyne-Beasley T, Aguilar-Gaxiola S. Aligning the goals of community-engaged research: why and how academic health centers can successfully engage with communities to improve health. Acad Med. 2012;87(3):285.

7. Fixsen DL, Naoom SF, Blase KA, Friedman RM. Implementation research: a synthesis of the literature. Tampa: University of South Florida, Louis de la Parte Florida Mental Health Institute, The National Implementation Research Network (FMHI Publication \#231); 2005.

8. Green LW. From research to "best practices" in other settings and populations. Am J Health Behav. 2001;25(3):165-78.

9. Dwyer J, Boulton A, Lavoie JG, Tenbensel T, Cumming J. Indigenous peoples' health care: new approaches to contracting and accountability at the public administration frontier. Public Manag Rev. 2014;16(8):1091-112.

10. Anderson I, Robson B, Connolly M, Al-Yaman F, Bjertness E, King A, Tynan M, Madden R, Bang A, Coimbra CE Jr. Indigenous and tribal peoples' health (The Lancet-Lowitja Institute Global Collaboration): a population study. Lancet. 2016;388:131-57.

11. Mark GT, Lyons AC. Maori healers' views on wellbeing: the importance of mind, body, spirit, family and land. Soc Sci Med. 2010;70:1756-64.

12. Lee CC, Armstrong KL. Indigenous models of mental health intervention: lessons from traditional healers. In: Ponterotto JG Casas JM, Suzuki LA, Alexander CM, editors. Handbook of multicultural counseling. Thousand Oaks: Sage Publications; 1995. p. 441-56

13. Angell BJ, Muhunthan J, Irving M, Eades S, Jan S. Global systematic review of the cost-effectiveness of indigenous health interventions. PloS One. 2014; 9:e111249. 
14. Wallerstein N, Duran B. Community-based participatory research contributions to intervention research: the intersection of science and practice to improve health equity. Am J Public Health. 2010;100(S1):S40-6

15. Oetzel J, Scott N, Hudson M, Masters-Awatere B, Rarere M, Foote J, Beaton A Ehau T. Implementation framework for chronic disease intervention effectiveness in Māori and other indigenous communities. Glob Health. 2017;13:69.

16. Gibson O, Lisy K, Davy C, Aromataris E, Kite E, Lockwood C, Riitano D, McBride K, Brown A. Enablers and barriers to the implementation of primary health care interventions for Indigenous people with chronic diseases: a systematic review. Implement Sci. 2015;10:71.

17. Dutta MJ. Communicating about culture and health: theorizing culturecentered and cultural sensitivity approaches. Commun Theory. 2007;17:304-28.

18. Basu A, Dutta MJ. Sex workers and HIV/AIDS: analyzing participatory culturecentered health communication strategies. Hum Commun Res. 2009;35:86-114.

19. Ramsden I, Spoonley P. The cultural safety debate in nursing education in Aotearoa. NZ Annu Rev Educ. 1994;3:161-74.

20. Dutta M, Anaele A, Jones C. Voice of hunger: addressing health disparities through the culture-centered approach. J Commun. 2013;63:159-80.

21. O'Mara-Eves A, Brunton G, McDaid D, Oliver S, Kavanagh J, Jamal F, et al. Community engagement to reduce inequalities in health: a systematic review, meta-analysis and economic analysis. Public Health Res. 2013:1:4

22. O'Mara-Eves A, Brunton G, Oliver S, Kavanagh J, Jamal F, Thomas J. The effectiveness of community engagement in public health interventions for disadvantaged groups: a meta-analysis. BMC Public Health. 2015;15:129.

23. Wallerstein N, Duran B, Oetzel JG, Minkler M, editors. Community-based participatory research for health: advancing social and health equity (3rd ed.). San Francisco: Jossey-Bass; 2018.

24. Yuen T, Park A, Seifer S, Payne-Sturges D. A systematic review of community engagement in the US Environmental Protection Agency's extramural research solicitations: implications for research funders. Am J Public Health. 2015;105:e44-52

25. CTSA Community Engagement Key Function Committee. Principles of community engagement, 2nd edition. Washington DC: NIH Publication No. 11-7782; 2011

26. Cook WK. Integrating research and action: a systematic review of community-based participatory research to address health disparities in environmental and occupational health in the USA. J Epidemiol Commun Health. 2008;62(8):668-76.

27. Wallerstein N, Oetzel JG, Duran B, Tafoya G, Belone L, Rae R. CBPR: What predicts outcomes? In: Minkler M, Wallerstein N, editors. Community based participatory research for health, 2nd ed. San Francisco: Jossey-Bass; 2008. p. 371-92.

28. Rittel HW, Webber MM. Dilemmas in a general theory of planning. Policy Sci. 1973:4(2):155-69.

29. Frerichs L, Lich KH, Dave G, Corbie-Smith $G$. Integrating systems science and community-based participatory research to achieve health equity. Am J Public Health. 2016;106:215-22.

30. Stephens A. Principled success: eco-feminism and systems thinking come together for better project outcomes. Int J Manag Proj Bus. 2013;6(1):199-209.

31. Carey G, Malbon E, Carey N, Joyce A, Crammond B, Carey A. Systems science and systems thinking for public health: a systematic review of the field. BMJ Open. 2015;5:e009002.

32. Wilkinson J, Goff M, Rusoja E, Hanson C, Swanson RC. The application of systems thinking concepts, methods, and tools to global health practices: An analysis of case studies. J Eval Clin Pract. 2018;24(3):607-18.

33. Grimshaw JM, Eccles MP, Lavis JN, Hill SJ, Squires JE. Knowledge translation of research findings. Implement Sci. 2012;7:50.

34. Strauss S, Tetroe J, Graham I. Defining knowledge translation. Can Med Assoc J. 2009;181:165-8.

35. Lavis J. Research, public policymaking, and knowledge-translation processes: Canadian efforts to build bridges. J Contin Educ Health. 2006;26:37-45.

36. Graham I, Logan J, Harrison M, Strauss S, Tetroe J, Caswell W, Robinson N. Lost in knowledge translation: time for a map? J Contin Educ Health. 2006;26:13-24.

37. Smylie J, Martin CM, Kaplan-Myrth N, Steele L, Tait C, Hogg W. Knowledge translation and indigenous knowledge. Int J Circumpolar Health. 2004;63(sup2):139-43.

38. Moher D, Liberati A, Tetzlaff J, Altman DG, Group P. Preferred reporting items for systematic reviews and meta-analyses: the PRISMA statement. PLoS medicine. 2009;6(7):e1000097.

39. Page M, McKenzie J, Higgins J. Tools for assessing risk of reporting biases in studies and syntheses of studies: a systematic review. BMJ Open. 2018;8:e019703.
40. Tipene-Leach DC, Coppell KJ, Abel S, Pāhau HL, Ehau T, Mann JI. Ngāti and healthy: Translating diabetes prevention evidence into community action. Ethn Health. 2013;18(4):402-14.

41. Blundell R, Gibbons V, Lillis S. Cultural issues in research, a reflection. N Z Med J. 2010;123(1309):97-105.

42. Joanna Briggs Institute. Critical appraisal tools. https://joannabriggs.org/ research/critical-appraisal-tools.html. Accessed 8 July 2019.

43. Wells S, Tamir O, Gray J, Naidoo D, Bekhit M, Goldmann D. Are quality improvement collaboratives effective? A systematic review. BMJ Qual Saf. 2017;27:226-40

44. Benyshek DC, Chino M, Dodge-Francis C, Begay TO, Jin H, Giordano C. Prevention of type 2 diabetes in urban American Indian/Alaskan Native communities: the Life in BALANCE pilot study. J Diabetes Mellitus. 2013; 3(4):184-91.

45. Christopher S, Gidley AL, Letiecg B, Smith A, McCormick AKHG. A cervical cancer community-based participatory research project in a Native American community. Health Educ Behav. 2008;35(6):821-34.

46. Kaholokula J, Wilson R, Townsend C, Zhang G, Chen J, Yoshimura S, Dillard A, Yokota J, Palakiko D, Gamiao S. Translating the diabetes prevention program in native Hawaiian and Pacific Islander communities: the PILI 'Ohana project. Transl Behav Med. 2014:4(2):149-59.

47. Shah VO, Carroll C, Mals R, Ghahate D, Bobelu J, Sandy P, Colleran K, Schrader R, Faber T, Burge MR. A home-based educational intervention improves patient activation measures and diabetes health indicators among Zuni Indians. PLoS ONE. 2015;10(5):e0125820.

48. Reilly RE, Cincotta M, Doyle J, Firebrace BR, Cargo M, van den Tol G, Morgan-Bulled D, Rowley KG. A pilot study of Aboriginal health promotion from an ecological perspective. BMC Public Health. 2011;11:749.

49. Coppell KJ, Tipene-Leach DC, Pahau HL, Williams SM, Abel S, lles M, Hindmarsh JH, Mann Jl. Two-year results from a community-wide diabetes prevention intervention in a high risk indigenous community: the Ngati and Healthy project. Diabetes Res Clin Pract. 2009;85(2):220-7.

50. Kakekagumick KE, Naqshbandi Hayward M, Harris SB, Saksvig B, Gittelsohn J, Manokeesic G, Goodman S, Hanley AJ. Sandy Lake Health And Diabetes Project: a community-based intervention targeting type 2 diabetes and its risk factors in a First Nations Community. Front Endocrinol. 2013:4:170.

51. Sinclair KA, Makahi EK, Shea-Solatorio C, Yoshimura SR, Townsend CK, Kaholokula JK. Outcomes from a diabetes self-management intervention for Native Hawaiians and Pacific People: partners in care. Ann Behav Med. 2012:45(1):24-32.

52. Kaholokula JK, Mau MK, Efird JT, Leake A, West M, Palakiko D-M, Yoshimura SR, Kekauoha BP, Rose C, Gomes H. A family and community focused lifestyle program prevents weight regain in Pacific Islanders: a pilot randomized controlled trial. Health Educ Behav. 2012;39(4):386-95.

53. Karanja N, Lutz T, Ritenbaugh C, Maupome G, Jones J, Becker T, Aickin M. The TOTS community intervention to prevent overweight in American Indian toddlers beginning at birth: a feasibility and efficacy study. Publ Health Promot Dis Prev. 2010;35(6):667-75.

54. Tomayko EJ, Prince RJ, Cronin KA, Adams AK. The Healthy Children, Strong Families intervention promotes improvements in nutrition, activity and body weight in American Indian families with young children. Public Health Nutr. 2016;19(15):2850-9.

55. Brimblecombe J, Ferguson M, Chatfield MD, Liberato SC, Gunther A, Ball K, Moodie M, Miles E, Magnus A, Mhurchu CNJTLPH. Effect of a price discount and consumer education strategy on food and beverage purchases in remote Indigenous Australia: a stepped-wedge randomised controlled trial. Lancet Public Health. 2017;2(2):e82-e95.

56. Canuto K, Cargo M, Li M, D'Onise K, Esterman A, McDermott RJBPH. Pragmatic randomised trial of a 12-week exercise and nutrition program for Aboriginal and Torres Strait Islander women: clinical results immediate post and 3 months follow-up. BMC Public Health. 2012;12:933.

57. Mendham AE, Duffield R, Marino F, Coutts AJ. A 12-week sports-based exercise programme for inactive Indigenous Australian men improved clinical risk factors associated with type 2 diabetes mellitus. J Sci Med Sport. 2015;18:438-43.

58. Ho LS, Gittelsohn J, Rimal R, Treuth MS, Sharma S, Rosecrans A, Harris SB. An integrated multi-institutional diabetes prevention program improves knowledge and healthy food acquisition in Northwestern Ontario First Nations. Health Educ Behav. 2008;35(4):561-73.

59. Kolahdooz F, Pakseresht M, Mead E, Beck L, Corriveau A, Sharma S. Impact of the Healthy Foods North nutrition intervention program on Inuit and 
Inuvialuit food consumption and preparation methods in Canadian Arctic communities. Nutr J. 2014;13:68.

60. Simmons D, Rush E, Crook N. Development and piloting of a community health worker-based intervention for the prevention of diabetes among New Zealand Maori in Te Wai o Rona: Diabetes Prevention Strategy. Public Health Nutr. 2008;11(12):1318-25.

61. English KC, Fairbanks J, Finster CE, Rafelito A, Luna J, Kennedy M. A socioecological approach to improving mammography rates in a tribal community. Health Educ Behav. 2008;35(3):396-409.

62. Townsend C, Dillard A, Hosoda K, Maskarinec G, Maunakea A, Yoshimura S, Hughes C, Palakiko D-M, Kehauoha B, Kaholokula J. Community-based participatory research integrates behavioral and biological research to achieve health equity for Native Hawaiians. Int J Environ Res Public Health. 2016;13(1):1-10

63. Tumiel-Berhalter L, Kahn L, Watkins R, Goehle M, Meyer C. The implementation of Good for the Neighborhood: a participatory community health program model in four minority underserved communities. Publ Health Promot Dis Prev. 2011;36(4):669-74.

64. Sushames A, Engelberg T, Gebel K. Perceived barriers and enablers to participation in a community-tailored physical activity program with Indigenous Australians in a regional and rural setting: a qualitative study. Int J Equity Health. 2017;16:172.

65. Oetzel JG, Villegas M, Zenone H, White Hat E, Wallerstein N, Duran B. Enhancing stewardship of community-engaged research through governance. Am J Public Health. 2015;105:1161-7.

66. Smith LT. Decolonizing methodologies: research and Indigenous peoples (2nd ed.). London: ZED Books; 2012.

67. Milton B, Attree P, French B, Povall S, Whitehead M, Popay J. The impact of community engagement on health and social outcomes: a systematic review. Commun Dev J. 2011:47(3):316-34

68. Durie M. Understanding health and illness: research at the interface between science and indigenous knowledge. Int J Epidemiol. 2004; 33(5):1138-43.

69. Barrera M, Castro F, Strycker L, Toobert D. Cultural adaptations of behavioral health interventions: a progress report. J Consult Clin Psychol. 2013;81 (2):196-205.

70. Kok MC, Dieleman M, Taegtmeyer M, Broerse JE, Kane SS, Ormel H, Tijm $\mathrm{MM}$, de Koning KA. Which intervention design factors influence performance of community health workers in low-and middle-income countries? A systematic review. Health Policy Plan. 2014;30(9):1207-27.

71. Jack HE, Arabadjis SD, Sun L, Sullivan EE, Phillips RS. Impact of community health workers on use of healthcare services in the United States: a systematic review. J Gen Intern Med. 2017;32:325-44

72. Hill J, Peer N, Oldenburg B, Kenge AP. Roles, responsibilities and characteristics of lay community health workers involved in diabetes prevention programmes: a systematic review. PloS One. 2017;12: e0189069.

73. Reilly R, Evans K, Gomersall J, Gorham G, Peters MDJ, Warren S, O'Shea R, Cass A, Brown A. Effectiveness, cost effectiveness, acceptibility and implementation barriers/enablers of chronic kidney disease managment programs for Indigenous people in Australia, New Zealand and Canada: a systematic review of mixed evidence. BMC Health Serv Res. 2016;16:119.

74. Gilmore B, MCAuliffe E. Effectiveness of community health workers delivering preventiv interventions for maternal and child health in low- and middleincome countries: a systematic review. BMC Public Health. 2013;13:847.

75. Roland KB, Milliken EL, Rohan EA, DeGroff A, White S, Melillo S, Rorie WE, Signes C-AC, Young PA. Use of community health workers and patient navigators to improve cancer outcomes among patients served by federally qualified health centers: a systematic literature review. Health Equity. 2017; 1(1):61-76.

76. Daellenbach HG, Flood RL. The informed student guide to management science: Cengage Learning EMEA; 2002.

77. Midgley G. Systemic intervention for public health. Am J Public Health. 2006;96(3):466-72.

78. Mignone J, Vargas JHG. Commentary: health care organization in Colombia: an Indigenous success story within a system in crisis. AlterNative: An International Journal of Indigenous Peoples. 2015;11(4):417-25.

\section{Publisher's Note}

Springer Nature remains neutral with regard to jurisdictional claims in published maps and institutional affiliations.

Ready to submit your research? Choose BMC and benefit from:

- fast, convenient online submission

- thorough peer review by experienced researchers in your field

- rapid publication on acceptance

- support for research data, including large and complex data types

- gold Open Access which fosters wider collaboration and increased citations

- maximum visibility for your research: over $100 \mathrm{M}$ website views per year

At BMC, research is always in progress.

Learn more biomedcentral.com/submissions 